\title{
Male Germ Cell Gene Expression
}

\author{
EDWARD M. EDDY \\ Laboratory of Reproductive and Developmental Toxicology, National Institute of Environmental \\ Health Sciences, National Institutes of Health, Research Triangle Park, North Carolina 27709
}

\begin{abstract}
Formation of the male gamete occurs in sequential mitotic, meiotic, and postmeiotic phases. Many germ cell-specific transcripts are produced during this process. Their expression is developmentally regulated and stage specific. Some of these transcripts are product of genes that are male germ cell-specific homologs of genes expressed in somatic cells, while some are expressed from unique genes unlike any others in the genome. Others are alternate transcripts derived from the same gene as transcripts in somatic cells but differing from them in size and/or overall sequence. They are generated during gene expression by using promoters and transcription factors that activate transcription at different start sites upstream or downstream of the usual site, by incorporation of alternate exons, by germ cell-specific splicing events, and by using alternate initiation sites for polyadenylation. Male germ cell development consists of an assortment of unique processes, including meiosis, genetic recombination, haploid gene expression, formation of the acrosome and flagellum, and remodeling and condensation of the chromatin. These processes are intricate, highly ordered, and require novel gene products and a precise and well-coordinated program of gene expression to occur.

The regulation of gene expression in male germ cells occurs at three levels: intrinsic, interactive, and extrinsic. A highly conserved genetic program "intrinsic" to germ cells determines the sequence of events that underlies germ cell development. This has been underscored by recent studies showing that meiosis involves many genes that have been conserved during evolution from yeast to man. During meiosis and other processes unique to germ cells, the intrinsic program determines which genes are utilized and when they are expressed. In the postmeiotic phase, it coordinates the expression of genes whose products are responsible for constructing the sperm. The process of spermatogenesis occurs in overlapping waves, with cohorts of germ cells developing in synchrony. The intrinsic program operating within a particular germ cell requires information from and provides information to neighboring cells to achieve this coordination. Sertoli cells are crucial for this "interactive" process as well as for providing essential support for germ cell proliferation and progression through the phases of development. The interactive level of regulation is dependent on "extrinsic" influences, primarily testosterone and follicle-stimulating hormone (FSH). Studies during the last 4 years have established that FSH is not essential for germ cell development but instead serves an important supportive role for this process. While testosterone is essential for maintenance of spermatogenesis, it acts on Sertoli cells and peritubular cells and has indirect effects on germ cells. The extrinsic and interactive processes are extremely important for establishing and maintaining an optimum environment within which gametogenesis occurs. Nevertheless, an intrinsic evolutionarily conserved genetic program regulates male germ cell gene expression and development.
\end{abstract}




\section{Introduction}

Spermatogenesis is a paradigm of development that continues throughout adult life in most mammals. This process occurs in seminiferous tubules containing an epithelium populated by a mixture of germ cells and Sertoli cells, surrounded by a thin wall of peritubular cells (Figure 1). Sertoli cells serve a crucial nurturing role for germ cells and are believed to help coordinate important events of spermatogenesis (Griswold, 1998). Sertoli cells also divide the seminiferous epithelium into two compartments: a basal compartment where cells are exposed to the surrounding milieu and a luminal compartment where cells are sequestered behind a "blood-testis" barrier formed by junctional complexes between Sertoli cells. Although the focus here is on the mouse, most of what is discussed applies to mammals in general.

Germ cell development occurs in successive mitotic, meiotic, and postmeiotic phases (Figure 2), with the germ cells moving from the periphery to the lumen of the seminiferous tubule during this process. The mitotic phase occurs in the basal compartment, while the meiotic and postmeiotic phases occur in the luminal compartment. This takes about 35 days in the mouse, with the mitotic phase lasting approximately 11 days, the meiotic phase lasting approximately 10

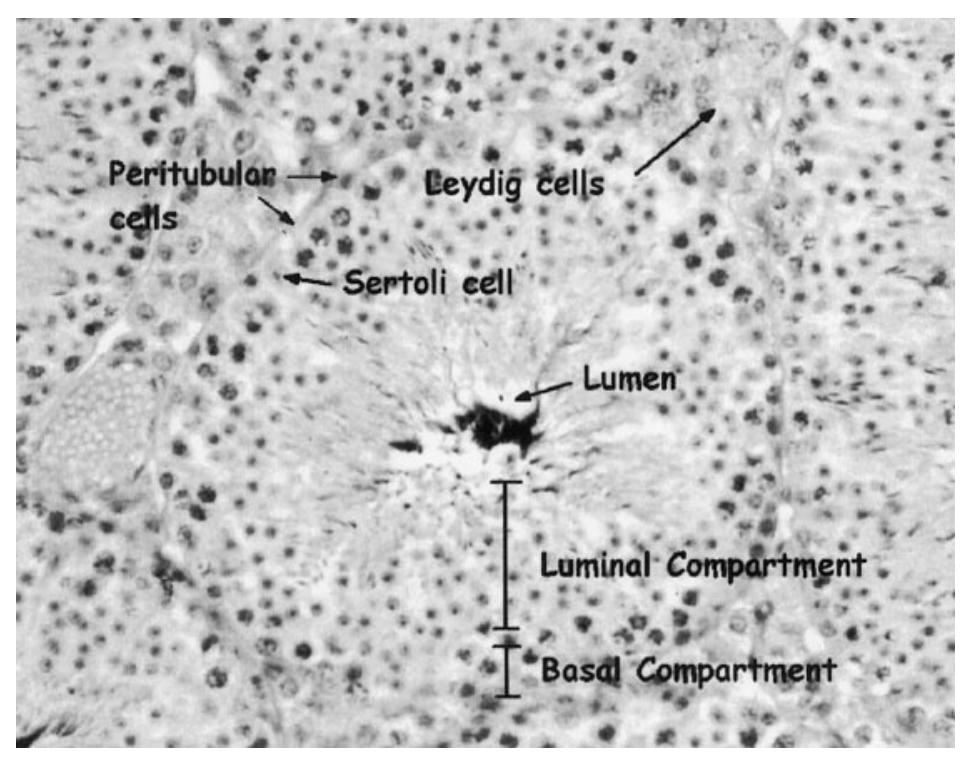

FIG. 1. A cross-section of a seminiferous tubule in the mouse testis. A thin layer of peritubular cells surrounds the tubule and Leydig cells are located in the areas between tubules. Sertoli cells divide the seminiferous tubule into the basal compartment containing germ cells in the mitotic phase and a luminal compartment containing germ cells in meiotic and postmeiotic phases. 


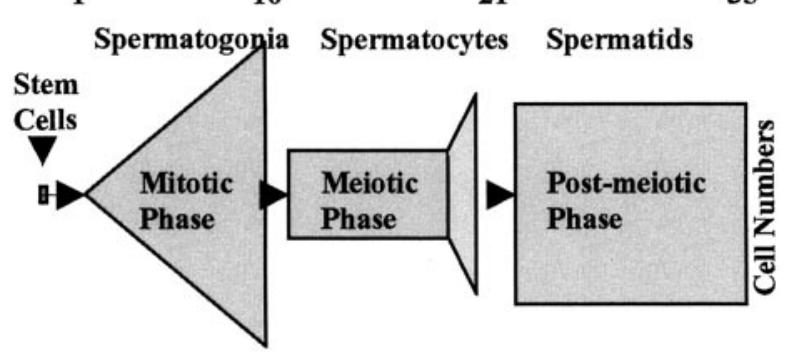

\section{Apoptosis Meiotic Divisions}

FIG. 2. The three phases of germ cell development. Stem cells give rise to spermatogonia that undergo several mitotic divisions over the next 10 days. More than half of the spermatogonia undergoes apoptosis during the latter part of the mitotic phase. During much of the meiotic phase, the spermatocytes are in the $\mathrm{G} 2$ phase of the cell cycle. After the two meiotic divisions at the end of this phase, germ cells enter the postmeiotic phase. Transcription occurs throughout germ cell development until the midpoint of the postmeiotic phase. During the latter half of this phase, proteins are synthesized from transcripts that have been stored since the early part of the postmeiotic phase.

days, and the postmeiotic phase lasting approximately 14 days (Clermont and Trott, 1969). The process begins every 8.7-8.9 days. The duration of these phases varies slightly between mouse strains. It includes processes unique to germ cells, including meiosis, genetic recombination, haploid gene expression, formation of the acrosome and flagellum, and remodeling and condensation of chromatin, all of which must occur at the correct time to produce the male gamete.

The highly ordered process of spermatogenesis, in turn, requires a precise and well-coordinated program that regulates the constantly changing patterns of gene expression. Although many questions remain to be answered, the regulation of this process can be thought of as occurring in three concentric levels: intrinsic, interactive, and extrinsic (Figure 3). Intrinsic regulation is managed by an evolutionarily conserved genetic program that underlies the development of germ cells of diverse species, ranging from nematodes to flies to mammals. This program is responsible for the sequence of events of differentiation and morphogenesis that occur throughout the progression of spermatogenesis. For meiosis and other processes specific to germ cells in particular, the intrinsic program determines which genes are utilized and when they are expressed.

The development of male germ cells occurs in overlapping waves in mammals and other vertebrates, with cohorts of mitotic, meiotic, and postmeiotic germ cells developing in synchrony. The intrinsic program operating within a given cell requires information from, and provides information to, neighboring cells to achieve this coordination. This interactive regulation of germ cell gene 


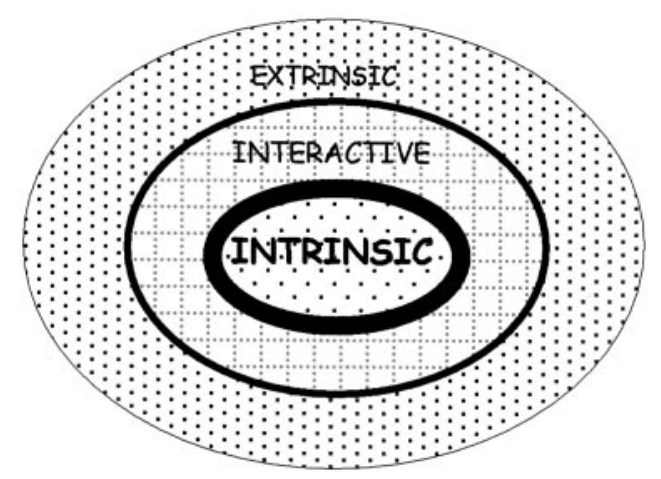

FIG. 3. The three levels of regulation of gene expression in germ cells. An intrinsic genetic program is responsible for regulating gene expression in male germ cells. Germ cells develop in overlapping waves, with cohorts of germ cells developing in synchrony. This requires coordination of the intrinsic programs for the cohorts of germ cells. The Sertoli cells serve a crucial role in this process. The interactive level of influence over germ cell development, in turn, is responsive to extrinsic cues that influence gene expression in Sertoli and Leydig cells, thereby regulating the milieu within which germ cells develop and indirectly influencing gene expression in germ cells. The extrinsic and interactive programs are essential for supporting germ cell development but gene expression is regulated by the germ cells themselves.

expression is far from being well understood but in vitro and in vivo studies indicate that Sertoli cells are key players in the information-exchange process. Each Sertoli cell is in contact simultaneously with three or four layers of germ cells that are in all three phases of spermatogenesis and is well situated to integrate and modulate the various signals to, from, and between germ cells.

Interactive regulation is, in turn, subject to extrinsic regulation by the endocrine system, primarily through testosterone and FSH. These hormones directly influence gene expression in somatic cells of the testis, thereby regulating the milieu within which interactive regulation occurs and indirectly influencing gene expression in germ cells.

\section{Intrinsic Regulation of Germ Cell Gene Expression}

The ultimate regulation of gene expression in male germ cells, as in all other cells, occurs first at the level of transcription, next at the level of translation, and thereafter at the post-translational level. The fundamental apparatus for these processes and the mechanisms that control them vary little between cell types but the specific features of the individual proteins involved and how their actions are modulated greatly influence the progression and outcome of the cell's development. There are three important features of the intrinsic genetic program underlying the process of male germ cell development. First, the processes of 
meiosis and spermatogenesis have been conserved throughout evolution. Second, many unique genes and variant transcripts are expressed during male germ cell development. Third, the expression of such genes is developmentally regulated and stage specific.

\section{A. GENETIC CONSERVATION}

Many of the general features of germ cell development are well conserved between phyla (Roosen-Runge, 1977). It is becoming increasingly clear that the same is true for the genes involved. The genome of the budding yeast, Saccharomyces cerevisiae, has been sequenced and the temporal profile of gene expression during sporulation, when meiosis occurs, has been determined (Chu et al., 1998). A subset of 164 genes induced in the early, middle, and later periods of meiosis was identified and a computer-based search was used to identify homologs, genes that have evolved from a common ancestor, in C. elegans, Drosophila, and mammals (Hwang et al., 2001). Over $70 \%$ of the yeast genes were found to have homologs in C. elegans, Drosophila, mice and/or humans, providing strong evidence that a substantial amount of the genetic program of meiosis is well conserved between phyla. More than half of these genes are represented by expressed sequence tags (ESTs) from mouse cDNA libraries that span the period from before until after meiosis in the female germ cell line (Hwang et al., 2001).

Expression of many of the same genes presumably occurs during meiosis in the male. However, systematic and comprehensive sequencing of cDNA libraries covering all phases of spermatogenesis needs to be done before this can be verified. There are many differences between oogenesis and spermatogenesis, with different genes being expressed during these processes. This was demonstrated in recent studies that evaluated the expression of testis-specific genes for C. elegans and Drosophila (Andrews et al., 2000; Reinke et al., 2000). On microarrays that contained nearly two thirds of the predicted genes for $C$. elegans, $11.8 \%$ were germline-enriched genes, of which $46 \%$ were expressed specifically in the male gonad, $18 \%$ in the female gonad, and 36\% in both (Reinke et al., 2000). Comparisons of ESTs generated by sequencing cDNA libraries of Drosophila found that nearly half of testis ESTs did not match with any nongonadal Drosophila EST sequences and about two thirds of those differed from ovary ESTs (Andrews et al., 2000). These studies indicate that several hundred male gonad-specific genes are expressed in C. elegans and Drosophila. It remains to be determined how many of these genes have homologs in mouse and/or human but the studies by Hwang et al. (2001) suggest that a majority of the genes are conserved.

The principal role of the intrinsic program in germ cell development was shown quite dramatically when rat spermatogonial stem cells were transplanted 
into the seminiferous tubules of the mouse testis. The rat stem cell gave rise to normal-appearing rat sperm (Clouthier et al., 1996) that developed at the same rate as in the rat testis, which is slower than in the mouse (Franca et al., 1998). These studies show that germ cells carry all of the information and instructions needed for their own development.

\section{B. GERM CELL-SPECIFIC TRANSCRIPTS}

It was estimated that up to 20,000 different transcripts are present in a given cell population (Zhang et al., 1997). Most of these are products of ubiquitously expressed "housekeeping genes" that are responsible for the basic structures and functions common to all cells, while fewer are responsible for the specific features and activities of the particular cell type. As of this writing, the NCBI Mouse UniGene database (http://www.ncbi.nlm.nih.gov/UniGene/Mm.Home. $\mathrm{html}$ ) contains 18,673 sequence clusters that include cDNAs from testis libraries, with 2701 sequence clusters identified as containing cDNAs specifically from spermatogonia, spermatocyte, or spermatid libraries. However, these numbers are probably inflated. Most of the ESTs are only 300 to 600 bp in length, very few have been confirmed by other methods to be expressed in testis or male germ cells, and it is likely that many are ubiquitously expressed genes. In addition, an unknown fraction consists of nonoverlapping ESTs from different regions of full-length cDNA sequences. On the other hand, there are undoubtedly many cDNAs in germ cell libraries that have not yet been sequenced and the short cDNA sequences that are determined probably do not identify variant transcripts.

There are three principal ways that male germ cell-specific transcripts and proteins are produced. The first is by expression of genes only in spermatogenic cells that are homologs of genes expressed in somatic cells, or in somatic cells and germ cells. The second is by expression of unique genes, those without significant similarity to any other gene in the genome. The third is by expression of variant transcripts. These are transcribed from genes also expressed in somatic cells but are often smaller or larger than their somatic cell counterparts and are the result of using one or more alternate transcript start sites, transcript splice sites, exons, or polyadenylation signals.

\section{Male Germ Cell-specific Gene Homologs}

Genes expressed only in male germ cells frequently are homologs of genes expressed in somatic cells (reviewed in Eddy and O'Brien, 1998). In some cases, a gene that is expressed in somatic cells is inactivated and a germ cell homolog is activated. An example of this is glyceraldehyde 3-phosphate dehydrogenase $(G a p d)$, a highly conserved gene for an essential enzyme in the glycolytic pathway. Gapd usually is considered to be a housekeeping gene and its mRNA is commonly used as a loading control on northern blots. However, this gene is 
inactivated during the meiotic phase and the spermatogenic cell-specific Gapds gene is activated early in the postmeiotic phase of male germ cell development (Mori et al., 1992; Welch et al., 1992). The amino acid sequence of GAPDS is over $70 \%$ identical to that of GAPD and contains the appropriate amino acids in the locations necessary to form the binding pocket for the $\mathrm{NAD}^{+}$cofactor and the thioester substrate-binding site. Although the exon-intron structure of mouse Gapds is similar to that of Gapd (Welch et al., 1995), GAPDS has a large proline-rich domain at the N-terminus that is not present in GAPD.

It seems inefficient for male germ cells to use a new gene instead of an existing one that encodes a nearly identical protein. However, there are often significant advantages for male germ cells to have their "own" genes. One is that a germ cell homolog may compensate for a gene that is inactivated in male germ cells. An example of this is the phosphoglycerate kinase-1 ( $P g k l)$ gene that encodes another essential enzyme in the glycolytic pathway. Pgkl is located on the $\mathrm{X}$ chromosome, which is inactivated during the meiotic phase of male germ cell development. However, the germ cell-specific $P g k 2$ gene is located on an autosome and is activated soon thereafter. The $P g k 2$ gene lacks introns and was hypothesized to have evolved as a functional retroposon of the X-linked $P g k 1$ gene (Boer et al., 1987; McCarrey and Thomas, 1987). Expression of the Pgk2 gene is restricted to germ cells in most eutherian mammals but is expressed ubiquitously or in a subset of somatic tissues and in germ cells of different marsupials. It was hypothesized that tissue-specific enhancer activity and tissuespecific demethylation led to the restriction of $P g k 2$ expression to germ cells, thereby maintaining glycolysis when $P g k l$ expression ceases due to $\mathrm{X}$ chromosome inactivation (McCarrey, 1994).

Another advantage of germ cell-specific genes is that the encoded protein may fulfill the same role as that of the somatic cell's product but also have other structural or functional properties that serve unique roles in male germ cells. For example, GAPDS is anchored to the fibrous sheath by the proline-rich $\mathrm{N}$-terminal domain that is absent in GAPD (Bunch et al., 1998). Furthermore, most of the other enzymes in the glycolytic pathway in postmeiotic germ cells and sperm also have unique structural or functional properties (reviewed in Eddy et al., 1994). Several are known to be concentrated in the principal piece (Bunch et al., 1998). Glycolytic enzymes in sperm cofractionate with sperm tail components (Mohri et al., 1965) and are associated as a complex (Storey and Kayne, 1978). The unique structural and functional features of the other glycolytic enzymes may serve a role similar to the proline-rich domain of GAPDS for assembling this complex. Molecular modeling studies indicate that important differences occur in specific amino acids surrounding the $\mathrm{NAD}^{+}$cofactor-binding pocket and glyceraldehyde 3-phosphate (G3P) substrate-binding pocket of GAPD and GAPDS (unpublished observations). Earlier investigators were unaware that sperm contain GAPDS rather than GAPD but observed that sperm were signif- 
icantly more vulnerable than other cells to damage by environmental chemicals structurally similar to G3P (Jones, 1978; Stevenson and Jones, 1985). However, glycolysis is ineffective in spermatids. Addition of glucose to spermatids in vitro leads to an increase in the concentration of intermediates upstream of GAPD in the glycolytic pathway, while those downstream of GAPD decrease, leading to a substantial drop in adenosine triphosphate (ATP) levels (Nakamura et al., 1982; Jutte et al., 1983). This is strikingly different from the situation in sperm, where glucose is required for fertilization to succeed (Hoppe, 1976; Fraser and Quinn, 1981; Cooper, 1984). Differences in molecular structure between GAPDS and GAPD may provide the ability for GAPDS to serve as the switch from inactive glycolysis in postmeiotic germ cells to active glycolysis in sperm (Eddy et al., 1994).

Activation of germ cell-specific gene expression may also occur without the associated inactivation of a homolog. An example of this occurs for two members of the 70-kDa heat-shock protein (HSP70) family. The HSP70 proteins are chaperones that assist other proteins to fold as they emerge from the ribosome, escort them through the cytoplasm, help them assemble into complexes, and facilitate the refolding of proteins partially denatured by heat or other stresses. The genes for most HSP70 proteins are expressed in all tissues, either constitutively or following induction by heat shock and other stresses. However, a major $70-\mathrm{kDa}$ protein is present in germ cells that migrates to the same position on two-dimensional (2-D) gels as the HSP70 protein induced by heat shock in 3T3 cells (Allen et al., 1988a,b). The protein is synthesized in spermatocytes (O'Brien, 1987) and was recognized on immunoblots with a pan-HSP70 antibody. The cDNA was cloned and the sequence was found to correspond to a region in a genomic clone (Hsp70-2) isolated previously (Zakeri et al., 1988). Northern and in situ hybridization analysis found that transcription began in the early part of the meiotic phase and immunoblot and immunohistochemical analysis determined that the protein was expressed shortly thereafter (Rosario et al., 1992).

Mice with a targeted mutation in the $H s p 70-2$ gene were produced to determine if the HSP70-2 protein has a unique role in germ cells. Male mice homozygous for the mutation are infertile (Dix et al., 1996a), demonstrating that HSP70-2 serves a different role than the other HSP70 proteins present in germ cells. Germ cells in the mitotic and meiotic phases were present in the testis but development was arrested in late pachytene spermatocytes, at the G2/M phase transition of the meiotic cell cycle. This transition occurs in all cells and requires Cdc2 kinase activity, acquired when a cyclin A or B regulatory subunit binds to the $\mathrm{Cdc} 2$ catalytic subunit and triggers changes in $\mathrm{Cdc} 2$ phosphorylation. This suggested that HSP70-2 is a molecular chaperone required for Cdc2 activation (Zhu et al., 1997). HSP70-2 was found to associate with Cdc2, but not with cyclin $\mathrm{B} 1$ or the cyclin B1/Cdc2 heterodimer, in germ cells of wild-type mice. 
While cyclin B1 and Cdc2 were present in germ cells of male mice homozygous for the Hsp70-2 mutation, they failed to assemble into heterodimers, lacked Cdc2 kinase activity, and did not undergo the changes in phosphorylation seen in wild-type mice. This suggested that HSP70-2 is required for Cdc2 to dimerize with cyclin B1 and become an active kinase in male germ cells. This was verified by showing that addition of recombinant HSP70-2 protein to a homogenate of germ cells from homozygous mutant mice restored the ability of $\mathrm{Cdc} 2$ to form heterodimers with cyclin B1 and to become an active kinase (Zhu et al., 1997). It was suggested that HSP70-2 enables Cdc2 to acquire the necessary conformation to form a heterodimer with cyclin $\mathrm{B} 1$, thereby providing the protein kinase activity necessary for the G2/M transition to occur. However, Cdc2 serves the same role at the G2/M transition during mitosis in somatic cells and of meiosis in female germ cells, in the absence of HSP70-2. Although it is not yet understood why HSP70-2 is required only in male germ cells to achieve the G2/M transition, it apparently serves a chaperone role that is specifically required in male germ cells and is not provided by homologs.

Transcription of $H s c 70 t$ also is activated in postmeiotic male germ cells without changes in expression of other HSP70 family genes. A monoclonal antibody against a protein spot extracted from $2 \mathrm{D}$ gels of isolated male germ cells recognized a $70-\mathrm{kDa}$ protein present only in spermatids. The protein had immunoblotting and peptide map characteristics similar to HSC70, a constitutively expressed member of the HSP70 family (Maekawa et al., 1989). The monoclonal antibody was used to screen a mouse testis cDNA library and clones were isolated with high sequence homology to an HSP70 family gene expressed only during the postmeiotic phase of spermatogenesis (Matsumoto and Fujimoto, 1990). Northern analysis demonstrated that Hsc70t transcripts are present throughout much of the postmeiotic phase, while immunostaining and immunblot analysis indicated that HSC70T protein is not synthesized until late in the postmeiotic phase (Tsunekawa et al., 1999).

It was hypothesized that HSC70T has a role similar to that of HSC70-2, serving as a chaperone for a protein or proteins of male germ cells essential during the late postmeiotic phase. The gene targeting approach was used to test this hypothesis. However, male mice homozygous for a mutation in $\mathrm{Hsc} 70 \mathrm{t}$ had normal fertility and histological studies indicated that germ cell development during the late postmeiotic phase was not altered (unpublished observations). Nevertheless, when sperm from homozygous mice were placed in vitro, they became immotile within an hour, while sperm from wild-type mice remained motile for several hours. ATP levels are low in these sperm and their ability to produce ATP appears to be compromised (E.M. Eddy, unpublished observations). This suggests that HSC70t possesses unique chaperone capabilities that are required by postmeiotic germ cells for the assembly and function of protein complexes involved in energy production. 
A variation on the theme of activating a gene without inactivating its homolog is seen for A-kinase anchoring proteins (AKAPs). They tether cAMPdependent protein kinase A (PKA), serving to localize its signal transduction activity within a particular region of the cell. The AKAPs differ substantially in sequence but all contain PKA-binding domains. Two AKAPs unique to male germ cells are located in the fibrous sheath, a cytoskeletal component of the sperm flagellum that contains at least six major proteins and more minor proteins in the mouse (Eddy et al., 1991). AKAP4 is the major structural protein of the fibrous sheath and the Akap4 gene is expressed only during the postmeiotic phase (Carrera et al., 1994; Fulcher et al., 1995). The Akap4 gene is located on the X chromosome, making it a single-copy gene in the male (Moss et al., 1997). The AKAP4 protein appears to serve as a scaffold for proteins that are tightly associated with the fibrous sheath or localized to the principal piece, including GAPDS. The Akap3 gene was identified recently and found to be expressed only during the postmeiotic phase (Mandal et al., 1999; Vijayaraghavan et al., 1999). The presence of two AKAPs in the fibrous sheath suggests that PKA activation and function lead to phosphorylation of proteins in this region that regulates flagellar function.

\section{Unique Genes}

Some genes expressed only in male germ cells do not have homologs in other cells. Most of these genes are expressed during the postmeiotic phase, when the specialized structural components of spermatozoa are produced. During this period, dramatic remodeling of the nucleus and condensation of the chromatin occurs, the acrosome forms, and the flagellar axoneme and accessory fiber proteins are synthesized and assembled.

The round spermatid nucleus is remodeled into a falciform-shaped nucleus in the mouse during the last part of the postmeiotic phase. This occurs by removal of the histones, the major nuclear proteins in all other cell types, and their replacement with transition proteins and those, in turn, with protamines. The transition proteins and protamines are products of unique genes expressed only in male germ cells. The protamines are small, basic proteins that package the sperm DNA into a volume about one twentieth of that of a nucleus of somatic cells (reviewed by Balhorn et al., 1999). Most mammals have only one protamine gene but mouse, human, and a few other mammals have two. Gene targeting was used to determine if both protamines are necessary or if protamine 2 (Prm2) is redundant to protamine 1 ( $\mathrm{Prml})$ in the mouse. One copy of either the Prml or Prm2 gene was disrupted in embryonic stem (ES) cells that were then injected into blastocysts to produce chimeras carrying the mutated gene. Although many male chimeras were produced containing a copy of the mutated Prm1 or Prm2 gene, no offspring heterozygous for the mutated gene were sired (Cho et al., 
2001). One possible explanation was that the chimeras did not produce sperm containing the mutated gene. The ES cells were derived from 129Sv strain mice and the blastocysts were from C57BL6 mice, which allowed the use of a polymerase chain reaction (PCR) assay based on microsatellite polymorphisms to genotype the sperm produced by chimeras. It was found that chimeras were producing 129-genotype sperm, of which half had a disrupted Prm1 or Prm2 gene. Subsequent studies determined that a decrease in either of the protamines disrupted sperm nuclear compaction, processing of protamine 2 from precursor to mature form, and normal sperm function. These changes occurred in all 129-genotype sperm, whether they had a copy of the intact gene or a copy of the mutated gene.

Although postmeiotic germ cells are haploid, spermatids remain interconnected by intercellular bridges and form a syncytium that allows sharing of mRNA and protein. It was concluded that transcript and/or protein sharing, coupled with lack of production of Prm1 or Prm2 in half of the spermatids, led to a reduction in amount of the protein in all of the spermatids within a syncytial cluster. This reduction compromised sperm development and function and caused the failure to transmit the intact or mutated Prm1 or Prm2 genes to offspring. These studies show that protamine 2 is not a functionally redundant protein and that both protamine 1 and protamine 2 are essential for the production of normally functioning sperm in the mouse (Cho et al., 2001).

Unique structures are present in germ cells during the meiotic phase, the major one being the synaptonemal complex. This structure, present in oocytes as well as spermatocytes, zippers together the homologous chromosomes to form the structural substrate upon which the processes of crossing over and genetic recombination occur. Its composition and function remain to be fully characterized but unique genes encode the SCP1 and COR1 proteins that are major structural components of the synaptonemal complex (Meuwissen et al., 1992; Dobson et al., 1994). The sex body or XY body is another unique structure present during meiosis in the male. It is located within the nucleus of spermatocytes and is the domain within which the $\mathrm{X}$ and $\mathrm{Y}$ chromosomes pair. Several antibodies have been used to identify proteins unique to the $\mathrm{XY}$ body (Kralewski and Benavente, 1997; Escalier and Garchon, 2000) but the identity of the genes has not been reported. Additional genes are expressed during meiosis that encode proteins involved in recombination and DNA repair but most are also expressed in somatic cells (reviewed in Eddy and O'Brien, 1998). However, the major phenotype for knockouts of several of these genes occurs in germ cells (reviewed in Eddy, 1999). The Dmcl gene is a homolog of yeast S. cerevisiae "disrupted in meiosis" gene and is expressed only during meiosis in male and female mice (Habu et al., 1996). A knockout of the Dmcl gene results in infertility due to failure of synapsis between homologous chromosomes during meiosis (Pittman et al., 1998; Yoshida et al., 1998). 


\section{Alternate Transcripts}

Male germ cell-specific proteins also may be synthesized from alternate transcripts that are derived from the same gene as transcripts in somatic cells but differ from them in size and/or overall sequence. They are generated during gene expression by using promoters and transcription factors that activate transcription at different start sites upstream or downstream of the usual site, by incorporation of alternate exons, by germ cell-specific splicing events, and by using alternate initiation sites for polyadenylation. Alternate transcripts in germ cells often are found serendipitously on northern blots as transcripts that are a different size in testis than in other cells.

Examples of alternate transcripts produced by several of these processes are seen for type 1 hexokinase. Hexokinase metabolizes glucose and is the first enzyme in the glycolytic pathway. Three germ cell-specific transcripts (Hkl-sa, $H k 1-s b, H k 1-s c)$ were identified. Genomic sequencing determined that the germ cell-specific $H k 1-s$ mRNAs in male mice are transcribed from the same gene as somatic cell $H k 1$ mRNA. The spermatogenic cell-specific domain replaced the $\mathrm{N}$-terminal mitochondrial porin-binding domain present in HK1 in somatic cells (Mori et al., 1993). The three cDNAs also differed from each other in the $5^{\prime}$ untranslated region. Northern analysis indicated that $H k 1$-sa transcripts are first present at low levels during meiosis, while the more abundant $H k 1$-s $b$ transcripts are detected only in postmeiotic germ cells. In addition, $H k l-s b$ contains a novel 23-residue sequence within the coding region that is not present in the Hkl-sa or $H k I$-sc sequences. Sequence analysis also showed that the common spermatogenic cell-specific sequence of the three $H k l-s$ transcripts was derived from two exons upstream of the exon encoding the porin-binding domain. Furthermore, the $5^{\prime}$ untranslated regions are found within other exons farther upstream (Mori et al., 1993). The HK1-S protein is present mainly within the principal piece region of the flagellum (Mori et al., 1998; Travis et al., 1998), the region where GAPDS is localized. Production of the three alternate transcripts for HK1-S involves utilization of germ cell-specific promoters, germ cell-specific transcription start sites, germ cell-specific exons, and alternate splice events (Mori et al., 1998).

Alternate transcripts also result from use of alternate polyadenylation sites in the 3' untranslated region. The cation-dependent mannose 6-phosphate receptor (CD-MPR) mediates transport of acid hydrolyases to lysosomes. Three transcripts for this receptor are present in spermatogenic cells. There is a 2.4-kb transcript comparable to that found in somatic cells, a $1.2-\mathrm{kb}$ transcript in pachytene spermatocytes, and a 1.4-kb transcript in round spermatids (O'Brien et al., 1994). The shorter transcripts in germ cells contain the full-length coding region present in the 2.4-kb transcript. By using probes for different regions of the 3'untranslated sequence on northern blots, it was found that the 1.4-kb and $1.2-\mathrm{kb}$ transcripts are produced by utilization of an alternate polyadenylation site. 
The difference in length of these two transcripts is due to a longer poly(A) tail on the 1.4-kb transcript (O'Brien et al., 1994).

Alternate transcripts are present in other cell types but are particularly abundant in male germ cells (reviewed in Eddy et al., 1993; Hecht, 1993; Eddy and O'Brien, 1998). There are at least four reasons that alternate transcripts may be beneficial to male germ cells. First, addition of new domains can expand the function of the protein and allow it to perform germ cell-specific functions. An example described previously is the proline-rich $\mathrm{N}$-terminal domain that tethers GAPDS to the fibrous sheath (Bunch et al., 1998). Second, removal of one domain and replacement with another can change the distribution of the protein within the cell. This occurs for HK-1 where the porin-binding domain that usually targets the enzyme to mitochondria is lost and a germ cell-specific domain is added (Mori et al., 1998), which presumably is responsible for HK1-S being located mainly in the principal piece. Third, a long poly(A) tail often is found on transcripts in postmeiotic germ cells for which there is a delay of several days between transcription and translation. These transcripts undergo shortening of the poly(A) tail coincident with becoming associated with polysomes and translationally active (Kleene et al., 1984). However, there are substantial differences in this process between various germ cell transcripts (Kleene, 1996) and it is unclear whether transcript shortening is a cause or an effect of translational activation of stored mRNAs. Fourth, alternate transcripts can arise by utilization of a promoter within an intron, resulting in a functionally different protein in male germ cells than in somatic cells. Examples of this are the truncated versions of angiotensin-converting enzyme (ACE) (Howard et al., 1990) and calcium ${ }^{+2} /$ calmodulin-dependent kinase IV (CaMKIV) (Means et al., 1991) that are found only in male germ cells and have enzymatic properties different from the longer forms.

\section{Developmentally Regulated Gene Expression}

The expression of many genes in male germ cells is developmentally regulated during the meiotic and postmeiotic phases. This probably is true for all germ cell-specific homologs, genes expressed only in male germ cells, and germ cell-specific transcripts. However, many other genes that are developmentally regulated in male germ cells are expressed in somatic cells as well (see reviews by Willison and Ashworth, 1987; Eddy et al., 1991,1993; Wolgemuth and Watrin, 1991; Hecht, 1993; Eddy and O'Brien, 1998).

Developmentally regulated gene expression in male germ cells may have evolved by more widely expressed genes becoming restricted in expression to male germ cells (McCarrey, 1994) or as the result of gene duplications or swapping of exons between precursor genes. In either case, these genes would need to incorporate promoters that drive their expression in male germ cells and 
not in other cells. Such promoters may have evolved from regulatory elements for meiosis-specific genes conserved from ancestral unicellular organisms. In addition, genes expressed in somatic cells also have undergone modifications to become developmentally regulated in germ cells. Two possible ways this may have occurred were either by incorporation of promoter elements regulating developmental expression in germ cells or by utilizing unique transcription factor combinations in germ cells that act through existing promoter elements. It is notable that gene-targeting studies involving genes expressed in somatic cells and germ cells often have found that the sole or major phenotype produced is in germ cells (reviewed in Eddy, 1999). A gene does not have to be expressed only in germ cells to be essential for germ cell development.

The regulation of transcription has not been studied in depth for any germ cell-specific genes, mainly because permanent germ cell lines are not available for use in conventional promoter-reporter assays. In lieu of this, transgenic mice have been used for promoter mapping for some germ cell-specific genes. Although this approach is cumbersome, the regions containing key regulatory elements for germ cell-specific and stage-specific gene expression have been delimited for several genes (Dix et al., 1996b; Iannello et al., 1997; Li et al., 1998; Reddi et al., 1999).

A diverse group of transcription factors are expressed in male germ cells. Some have been implicated in the transcriptional regulation of genes in specific stages of spermatogenesis (see Famia et al., 1999; Daniel and Habener, 2000; Liu et al., 2000; Han et al., 2001; and references therein). However, experimental evidence is available for only a few demonstrating a specific role in germ cells. One example is the Erg4 transcription factor. The only effect of a targeted mutation in the Erg4 gene was disruption of male germ cell development during the meiotic phase (Tourtellotte et al., 1999). Also, a knockout of the gene for SPRM-1, a POU-homeodomain gene expressed only in male germ cells during the postmeiotic phase, produced subnormal fertility (Pearse et al., 1997). In addition, a germ cell-specific subunit of the general transcription factor TFIIA was found to be upregulated in pachytene spermatocytes, along with several RNA polymerase II and III factors (Han et al., 2001). This coordinated expression of general transcription factor genes was hypothesized to regulate a particular subset of genes in germ cells (Han et al., 2001). An interesting parallel is that the general transcription factor TFIID is required for high level transcription of a set of stage-specific and tissue-specific target genes during male germ cell development in Drosophila (Hiller et al., 2001).

It has been recognized for nearly 20 years that activation of expression of some genes occurs specifically during the meiotic or postmeiotic phases. More recent studies indicate that developmentally regulated gene expression also occurs during the mitotic phase. The use of library subtraction methods to identify cDNAs present in a mouse primitive type A spermatogonia library led to 
the identification of 19 novel genes expressed only in the testis (Wang et al., 2001). Seven of these appeared to be involved in transcriptional or posttranscriptional regulation of gene expression. A relatively high fraction of these genes mapped to the $\mathrm{X}$ chromosome. It was suggested that the $\mathrm{X}$ chromosome has a pre-eminent role in male germ cell development that was acquired as the $\mathrm{X}$ chromosome evolved from an autosome (Wang et al., 2001).

\section{Interactive Regulation of Germ Cell Gene Expression}

Sertoli cells are essential for germ cells to proliferate and to progress through the phases of development. The general functions of Sertoli cells in these processes are well known, such as dividing the seminiferous epithelium into basal and luminal compartments and providing a suitable milieu within which germ cell development occurs. In addition, Sertoli cells are more amenable to in vitro study than germ cells. This has allowed the identification of a variety of proteins that are synthesized and exported by Sertoli cells, at least some of which are involved in the interaction with germ cells (Griswold, 1995,1998). However, what role most of these proteins serve in regulating germ cell development remains to be determined.

The relationship between germ cells and Sertoli cells is bi-directional. The patterns of gene expression in Sertoli cells change as neighboring germ cells progress through the stages of spermatogenesis (Zabludoff et al., 2001). This suggests that one aspect of germ cell-Sertoli cell interactions involves diverse signals coming from the associated cohort of germ cells to act on Sertoli cells and cause changes in gene expression, which alters feedback signals that modulate the intrinsic regulatory program driving germ cell development.

\section{A. SERTOLI CELL EFFECTS ON GERM CELLS}

Sertoli cells secrete more than 100 different proteins detectable by 2-D gel electrophoresis of conditioned media from primary Sertoli cell cultures (see reviews by Griswold, 1993,1998; Jégou, 1993; Dupaix et al., 1996), and seminiferous tubule fluid has a 10 -fold higher potassium concentration than blood (Hinton and Setchell, 1993). Sertoli cell products for which the identity is known include transport or binding proteins, proteases, energy metabolites, components of the extracellular matrix and junctional complexes, and a variety of factors that may influence germ cell division, differentiation, and metabolism (Jégou, 1993). The factors include several cytokines, a variety of growth factors, signaling molecules, bioactive peptides, and hormones (Griswold, 1993; Skinner, 1993). Although assumed to be involved in signaling from Sertoli to germ cell, experimental evidence demonstrating effects on gene expression in germ cells has been shown for only a few. Gene knockout studies have provided evidence 
that two factors produced by Sertoli cells - desert hedgehog (Dhh) (Bitgood et al., 1996) and glial cell line-derived neurotrophic factor (GDNF) (Meng et al., 2000) - are involved in signaling.

One of the receptors known to participate in Sertoli-to-germ-cell signaling is the bi-functional insulin-like growth factor-II/cation-independent mannose 6-phosphate (IGF-II/M6P) receptor. This receptor is present on the surface of spermatogenic cells and Sertoli cells secrete ligands for the receptor, including IGF-II and 10 or more M6P-bearing glycoprotein ligands that remain to be characterized (O'Brien et al., 1993; Tsuruta and O'Brien, 1995). Furthermore, proliferin, leukemia inhibitory factor (LIF), and the precursor forms of transforming growth factor beta (TGF $\beta$ ) are M6P-glycoprotein-containing growth factors found in the testis and may act through the IGFII/M6P receptor on germ cells. Addition of either IGF-II or of M6P-glycoproteins affinity-purified from Sertoli cell-conditioned medium to isolated spermatogenic cells produces dosedependent increases in mRNA for the immediate-early gene $c$-fos and in rRNA (Tsuruta and O'Brien, 1995; Tsuruta et al., 2000). This effect is seen in spermatogonia (Tsuruta et al., 2000), spermatocytes, and round spermatids (Tsuruta and O'Brien, 1995), indicating that interactions can occur between M6P-containing ligands and germ cells in both the basal and luminal compartments of the seminiferous epithelium, with the possibility that different ligands are secreted into each compartment.

Another example of Sertoli-to-germ-cell signaling is provided by stem cell factor (SCF) ligand and c-kit receptor interaction. The c-kit trans-membrane tyrosine kinase receptor is present on spermatogonia (Manova et al., 1990; Yoshinaga et al., 1991). Sertoli cells produce SCF (Rossi et al., 1991) and a mutation in the Kit gene or in Kitl, the gene for SCF, disrupts male germ cell development (reviewed in Besmer et al., 1993). SCF exists in two forms, membrane-associated and soluble, and Sertoli cells predominantly produce the membrane-associated form. This suggests that Sertoli cells communicate directly with spermatogonia through the association between SCF ligand and c-kit receptor.

\section{B. GERM CELL EFFECTS ON SERTOLI CELLS}

Addition of germ cells to Sertoli cell primary cultures can stimulate or inhibit different Sertoli cell functions (Jégou, 1993; Dupaix et al., 1996). Some of the germ cell factors that affect Sertoli cells are known to be proteins but have not been identified. In addition, meiotic and postmeiotic germ cells produce the growth factors $\beta \mathrm{NGF}$ (nerve growth factor), bFGF (fibroblast growth factor), IGF-I, and TGF $\beta$; and the cytokines interleukin-1 (IL-1), interferon (INF), and tumor necrosis factor alpha (TNF $\alpha$ ) (reviewed in Skinner, 1993; Dupaix et al., 1996; Gnessi et al., 1997) that are likely to signal Sertoli cells. A gene trap 
strategy to identify genes involved in signaling from germ cells to Sertoli cells found that Fral mRNA was induced in Sertoli cells by addition of either round spermatids or NGF to Sertoli cells. The NGF effect was mediated by the TrkA receptor and the ERK1-ERK2 kinase pathway (Vidal et al., 2001). In addition, mRNA differential display studies identified four genes that were upregulated and three genes that were downregulated in primary Sertoli cells when cocultured for 24 hours with germ cells. The same studies found that three genes were downregulated in germ cells in the cocultures, demonstrating the reciprocal nature of Sertoli and germ cell interactions (Syed and Hecht, 1997).

\section{GERM CELL EFFECTS ON GERM CELLS}

The effects of one population of germ cells on another is probably the least understood and certainly the least studied of the interactive regulatory processes of germ cell development. However, the results of loss-of-function and gain-offunction studies provide indirect evidence of communication between different populations of germ cells.

The bone morphogenetic protein 8B (BMP8B) is a member of the TGF $\beta$ growth factor superfamily and shows bimodal expression in developing germ cells. It is expressed in spermatogonia at 1 week after birth but not in those cells after BMP8A expression begins in round spermatids (Zhao et al., 1996). A knockout of the $B m p 8 b$ gene caused two separate effects on gene cells: a failure or reduction in proliferation that coincides with the early BMP8B expression and delayed differentiation, causing a reduction in mitotic and meiotic germ cells. BMP8B may act through two receptors that bind BMPs, ActRIIA, expressed mainly in spermatogonia and early meiotic cells, and ActRIIB, expressed mainly in pachytene spermatocytes and round spermatids. It was hypothesized that BMP8B produced by round spermatids acts on primary spermatocytes to regulate their survival and differentiation (Zhao et al., 1998).

The Bcl-2 family of proteins can have either positive or negative effects on the regulation of apoptosis (Korsmeyer, 1995). BAX forms a heterodimer with Bcl-2 and counters the apoptosis-repressing role of Bcl-2, thereby promoting apoptosis. BAX is expressed at high levels in spermatogonia (Rodriguez et al., 1997). Bax knockout mice are infertile, have atrophic testes, and lack sperm in the epididymis (Knudson et al., 1995). The substantial increase in apoptosis that occurs near the end of the mitotic phase is suppressed in Bax knockout mice. The increased number of spermatogonia has a detrimental effect on germ cells in other phases of spermatogenesis. Furthermore, transgenic mice that express high levels of the apoptosis-inhibiting proteins Bcl-2 or BclxL in spermatogonia also have increased numbers of spermatogonia and disrupted spermatogenesis (Furuchi et al., 1996; Rodriguez et al., 1997). In another study using the genetrapping procedure, a mutation was produced in the gene for Bclw, an apoptosis- 
inhibiting protein expressed during the postmeiotic phase (Ross et al., 1998). This resulted in a block in germ cell development in late spermatids in juvenile mice, followed by an increase in apoptosis in spermatocytes, which led to the loss of spermatids and degeneration of the seminiferous epithelium. In each of these transgenic or knockout mice, spermatogenesis was most affected in a phase of germ cell development different from the phase when expression of a Bcl-2 family protein was altered. A common feature of these studies is that the ratios between germ cells in mitotic, meiotic, and postmeiotic phases of germ cell development were altered. This suggests that an imbalance in reciprocal signals between germ cells that coordinate their development leads to disruption of spermatogenesis.

\section{BIOACTIVE PEPTIDES}

The genes for many bioactive peptides, originally considered to be neuroendocrine regulators, as well as the genes for many of their receptors are expressed in the testis. The peptides are produced in Leydig cells, Sertoli cells, peritubular myoid cells, and germ cells, and often in more than one cell type. Germ cells have been reported to have RNA or protein for the proenkephalin (pENK) pituitary adenylate cyclase-activating peptide (PACAP), pro-opiomelanocortin (POMC), and gonadotropin-releasing hormone $(\mathrm{GnRH})$ peptides and gastrinrelated peptide (GRP) receptor (reviewed by Gnessi et al., 1997). A larger number of peptides and receptors are present in the other cell types, perhaps suggesting a major role of the peptides and receptors in coordinating the activities of Leydig cells, Sertoli cells, and peritubular cells, and a lesser role in germ cell interactions. However, germ cells contain RNA or protein for bFGF, IGF-I, $\beta$-NGF and TGF growth factors, and the receptors for epidermal growth factor (EGF)/TGF $\alpha$, bFGF, stem cell factor (SCF), and IGF-I. This might indicate that growth factors have a greater role than bioactive peptides in regulating germ cells. The presence of growth hormone releasing hormone (GHRH), growth hormone (GH), IGF-I, and IGF binding proteins in the testis suggests an intra-testicular GH axis (DiMeglio et al., 1998). It is quite likely that these and probably other peptides and receptors are part of the intratesticular network that coordinates the biological activities of the various cell types but how significant a role they play remains to be determined.

\section{Extrinsic Regulation of Germ Cell Gene Regulation}

The dogma has been that FSH and testosterone regulate spermatogenesis, sometimes resulting in the view that germ cell development is a black box, with hormones going in one end and sperm coming out the other end. However, it has become increasingly apparent that FSH and testosterone do not regulate germ 
cell development but instead serve an important supportive role for this process. FSH is not essential for spermatogenesis, as shown by the findings that male mice with a knockout of the gene for FSH- $\beta$ are fertile (Kumar et al., 1997) and that men with an inactivating mutation in the FSH receptor had varying degrees of spermatogenic failure but were not infertile (Tapanainen et al., 1997). In addition, testis weight and epididymal sperm numbers were reduced in FSH receptor knockout mice (Krishnamurthy et al., 2000) but males were fertile. It has become apparent in the last 4 years that germ cell development is considerably less dependent on FSH than was previously thought.

The other major player in the extrinsic regulation of germ cell development, testosterone, is essential for this process. LH regulates the production of testosterone by Leydig cells and testosterone acts through androgen receptors (ARs) in Sertoli cells and peritubular cells to influence the Sertoli cell functions that support germ cell development (reviewed by Sar et al., 1993). In the absence of LH, spermatogenesis can be maintained by administering testosterone but not quite at the same level as in the normal state (reviewed by Sharpe, 1994). There are several studies indicating that androgen receptors are present in germ cells and several indicating that they are not. However, it has been shown recently that if there are ARs in germ cells, they are not essential for germ cell development. Using the germ cell transplantation technique, germ cells from the testis of Tfm mice lacking functional androgen receptors but carrying a $L a c Z$ transgene were transplanted into the testes of mice with functional ARs. Colonies of donor-derived spermatogenic cells were seen in the seminiferous tubules of recipient mice, indicating that mouse germ cells do not require functional ARs to carry out spermatogenesis (Johnston et al., 2001).

Not all extrinsic regulators of germ cell development act through Sertoli cells. Vitamin A is required for spermatogenesis. Vitamin A-deficient rats have only spermatogonia and Sertoli cells in their seminiferous tubules. However, treatment with retinol restores spermatogenesis in these animals (reviewed by Kim and Akmal, 1996). The retinoid acid receptor (RAR) $\alpha$ is present in spermatogenic cells (Wang and Kim, 1993) and the retinoid X receptor (RXR) $\beta$ is present in Sertoli cells (Kastner et al., 1996). These are members of the steroid receptor superfamily and gene knockout studies have demonstrated that each is essential for spermatogenesis (Lufkin et al., 1993; Kastner et al., 1996), strongly suggesting that vitamin A may have direct effects on germ cell development and function.

\section{Conclusions}

There is strong evidence that regulation of gene expression in male germ cells is largely an independent process. Male germ cells carry their own marching orders that have been refined and handed down over the millennia. Given the appropriate environment, spermatogonial stem cells can do their job of producing 
spermatogonia and/or other stem cells. The spermatogonia proliferate, then squeeze through a bottleneck where many die but some become spermatocytes. These cells carry out the complex process of meiosis, an ancient series of events that is good for the species but a dangerous prospect for the cell because of the intentional DNA damage that occurs during recombination. The next hurdle is for these cells to divide. Cell division is not an unusual process but germ cells make it complicated by dividing twice without making new copies of their chromosomes.

The postmeiotic cells are responsible for synthesizing and assembling a host of novel proteins that make up the sperm. Hidden away in the genome is a curious group of genes that are quiescent through most of the lifecycle of the organism. Only in spermatids do these genes become activated to carry out the construction of a hydrodynamically specialized cell containing a payload of tightly packaged DNA, an elaborate propulsion system, and special enzymes that help deliver the DNA to the egg. Most, if not all, of these processes are the responsibility of a program that operates without outside direction. Of course, germ cells need considerable help from other cells for all of this to happen and they are dependent on a remarkably complex support system. There is a hierarchy of local to distant systems responsible for ensuring that germ cells have the appropriate environment for producing the highly specialized cells necessary for continuation of the next and future generations of the species.

Sertoli cells in particular provide essential and highly effective support that is responsive and highly interactive, but not directive, for gene expression in male germ cells. The major functions of the Sertoli cells are heavily influenced by and dependent on other cells, particularly their neighbors, the peritubular myoid cells and Leydig cells. The functions of Sertoli cells are also modulated directly and indirectly by $\mathrm{FSH}, \mathrm{LH}$, and other hormones, whose influences range from crucial to beneficial at different times during the development and function of the seminiferous tubules. Although these extrinsic signals are of immeasurable importance for establishing and maintaining the environment within which male germ cells develop, it needs to remembered that they support, rather than regulate, male germ cell development.

\section{REFERENCES}

Allen RL, O'Brien DA, Jones CC, Rockett DL, Eddy EM 1988a Expression of heat shock proteins by isolated mouse spermatogenic cells. Mol Cell Biol 8:3260-3266

Allen RL, O'Brien DA, Eddy EM 1988b A novel hsp70-like protein (P70) is present in mouse spermatogenic cells. Mol Cell Biol 8:828-832

Andrews J, Bouffard G, Cheadle C, Lu J, Becker K, Oliver B 2000 Gene discovery using computation and microarray analysis of transcription in the Drosophila melanogaster testis. Genome Res 10:2030-2043 
Balhorn R, Cosman M, Thornton K, Krishnan V, Corzett M, Bench G, Kramer C, Lee J IV, Hud N, Allen M, Priety M, Meyer-IIse W, Brown J, Kirz J, Zhang X, Bradbury E, Maki G, Braun R, Breen W 1999 Protamine mediated condensation of DNA in mammalian sperm. In: Gagnon C, ed. The Male Gamete: From Basic Science to Clinical Applications. Vienna, IL: Cache River Press; 55-70

Besmer P, Manova K, Duttlinger R, Huang EJ, Packer A, Hyssler C, Bachvarova RF 1993 The kit-ligand (steel factor) and its receptor $c$-kit/W: pleiotropic roles in gametogenesis and melanogenesis. Development (suppl):125-137

Bitgood MJ, Shen L, McMahon AP 1996 Sertoli cell signaling by Desert hedgehog regulates the male germline. Current Biol 5:298-304

Boer PH, Adra CN, Lau Y-F, McBurney MW 1987 The testis-specific phosphoglycerate kinase gene pgk-2 is a recruited retroposon. Mol Cell Biol 7:3107-3112

Bunch DO, Welch JE, Magyar PL, Eddy EM, O'Brien DA 1998 Glyceraldehyde 3-phosphate dehydrogenase-S protein distribution during mouse spermatogenesis. Biol Reprod 58:834841

Carrera A, Gerton GL, Moss SB 1994 The major fibrous sheath polypeptide of mouse sperm: structural and functional similarities to the A-kinase anchoring proteins. Dev Biol 165:272284

Cho C, Willis W, Goulding EH, Jung-Ha H, Choi Y-C, Hecht NB, Eddy EM 2001 Haploinsufficiency of protamine-1 or -2 causes infertility in mice. Nature Genet 28:82-86

Chu S, Dervan PA, Botstein DBP, Herskowitz I 1998 The transcriptional program of sporulation in budding yeast. Science 282:699-705

Clermont Y, Trott M 1969 Duration of the cycle of the seminiferous epithelium in the mouse and hamster determined by means of 3H-thymidine and radioautography. Fertil Steril 20:805-817

Clouthier DE, Averbock MA, Maika SD, Hammer RE, Brinster RL 1996 Rat spermatogenesis in mouse testis. Nature 381:418-421

Cooper TG 1984 The onset and maintenance of hyperactivated motility of spermatozoa in the mouse. Gamete Res 9:55-74

Daniel PB, Habener JF 2000 Pituitary adenylate cyclase-activating polypeptide gene expression regulated by a testis-specific promoter in germ cells during spermatogenesis. Endocrinology 141:1218-1227

DiMeglio LA, Steinmetz R, Pescovitz OH 1998 Variations on a theme: testis-derived neuropeptide hormones. Eur J Endocrinol 139:361-362

Dix DJ, Allen JW, Collins BW, Mori C, Nakamura N, Poorman-Allen P, Goulding EH, Eddy EM 1996a Targeted gene disruption of Hsp70-2 results in failed meiosis, germ cell apoptosis, and male infertility. Proc Natl Acad Sci USA 93:3264-3268

Dix DJ, Rosario-Herrle M, Gotoh H, Mori C, Goulding EH, Barrett CV, Eddy EM 1996b Developmentally regulated expression of $H s p 70-2$ and a $H s p 70-2 / l a c Z$ transgene during spermatogenesis. Dev Biol 174:310-321

Dobson MJ, Pearlman RE, Karaiskakis A, Spyropoulos B, Moens PB 1994 Synaptonemal complex proteins: occurrence, epitope mapping and chromosome disjunction. J Cell Sci 107:2749-2760

Dupaix A, Pineau C, Piquet-Pellorce C, Jégou B 1996 Paracrine and autocrine regulations of spermatogenesis. In: Hamamah S, Mieusset R, eds. Research in Male Gametes: Production and Quality. Rennes, France: INSERM; 47-63

Eddy EM 1999 The effects of gene knockouts on spermatogenesis. In: Gagnon C, ed. The Male Gamete: From Basic Science to Clinical Applications. Vienna, IL: Cache River Press; 23-36

Eddy EM, O'Brien DA 1994 The spermatozoon. In: Knobil E, Neill JD, eds. The Physiology of Reproduction. New York: Raven Press; 29-77 
Eddy EM, O’Brien DA 1998 Gene expression during mammalian meiosis. In: Handel MA, ed. Meiosis and Gametogenesis. San Diego: Academic Press; 141-200

Eddy EM, O'Brien DA, Fenderson BA, Welch JE 1991 Intermediate filament-like proteins in the fibrous sheath of the mouse sperm flagellum. Ann NY Acad Sci 637:224-239

Eddy EM, Welch JE, O'Brien DA 1993 Gene expression during spermatogenesis. In: De Kretser DM, ed. Molecular Biology of the Male Reproductive System. Orlando, FL: Academic Press; 181-232

Eddy EM, Welch JE, Mori C, Fulcher KD, O'Brien DA 1994 Role and regulation of spermatogenic cell-specific gene expression: enzymes of glycolysis. In: Bartke A, ed. Function of Somatic Cells in the Testis. New York: Springer-Verlag; 362-372

Escalier D, Garchon H-J 2000 XMR is associated with the asynapsed segments of sex chromosomes in the XY body of mouse primary spermatocytes. Chromosoma 109:259-265

Famia G, De Casare D, Sassone-Corsi P 1999 CBP-independent activation of CREM and CREB by the LIM-only protein ACT. Nature 398:165-169

Franca LR, Ogawa T, Averbock MR, Brinster RL, Russell LD 1998 Germ cell genotype controls cell cycle during cell cycle during spermatogenesis in the rat. Biol Reprod 59:1371-1377

Fraser LR, Quinn PJ 1981 A glycolytic product is obligatory for initiation of the sperm acrosome reaction and whiplash motility required for fertilization in the mouse. J Reprod Fertil 61:25-35

Fulcher KD, Mori C, Welch JE, O'Brien DA, Klapper DG, Eddy EM 1995 Characterization of Fsc1 cDNA for a mouse sperm fibrous sheath component. Biol Reprod 52:41-49

Furuchi T, Masuko K, Nishimune Y, Obinata M, Matsui Y 1996 Inhibition of testicular germ cell apoptosis and differentiation in mice misexpression Bcl-2 in spermatogonia. Development 122:1703-1709

Gnessi L, Fabbri A, Spera G 1997 Gonadal peptides as mediators of development and functional control of the testis: an integrated system with hormones and local environment. Endocr Rev 18:541-609

Griswold MD 1993 Protein secretion by Sertoli cells: general considerations. In: Russell LD, Griswold MD, eds. The Sertoli Cell. Clearwater, FL: Cache River Press; 195-200

Griswold MD 1995 Interactions between germ cells and Sertoli cells in the testis. Biol Reprod 52:211-216

Griswold MD 1998 The central role of Sertoli cells in spermatogenesis. Semin Cell Dev Biol 9:411-416

Habu T, Taki T, West A, Nishimune Y, Morita T 1996 The mouse and human homologs of DMC1, the yeast meiosis-specific homologous recombination gene, have a common unique form of exon-skipped transcripts in meiosis. Nucleic Acids Res 24:470-477

Han S, Zhou L, Upadhyaya A, Lee S, Parker K, DeJong J 2001 TFII $\alpha / \beta$-like factor is encoded by a germ cell-specific gene whose expression is up-regulated with other general transcription factors during spermatogenesis in the mouse. Biol Reprod 64:507-517

Hecht NB 1993 Gene expression during male germ cell development. In: Desjardins C, Ewing LL, eds. Cell and Molecular Biology of the Testis. Oxford: Oxford University Press; 464-503

Hiller M, Lin T, Wood C, Fuller M 2001 Developmental regulation of transcription by a tissue-specific TAF homolog. Genes Dev 15:1021-1030

Hinton B, Setchell B 1993 Fluid secretion and movement. In: Russell LD, Griswold MD, eds. The Sertoli Cell. Clearwater, FL: Cache River Press; 249-267

Hoppe PC 1976 Glucose requirement for mouse sperm capacitation in vitro. Biol Reprod 15:39-45

Howard TE, Shai S-Y, Langford KG, Martin BM, Bernstein KE 1990 Transcription of testicular angiotensin-converting enzyme (ACE) is initiated within the 12th intron of the somatic ACE gene. Mol Cell Biol 10:4294-4302 
Hwang SY, Oh B, Knowles BB, Solter D, Lee JS 2001 Expression of genes involved in mammalian meiosis during the transition from egg to embryo. Mol Reprod Dev 59:144-158

Iannello R, Young J, Sumarsono S, Tymms M, Dahl H-H, Gould J, Hedger M, Kola I 1997 Regulation of Pdha-2 expression is mediated by proximal promoter sequences and $\mathrm{CpG}$ methylation. Mol Cell Biol 17:612-619

Jegou B 1993 The Sertoli-germ cell communication network in mammals. Intl Rev Cytol 147:25-96

Johnston DS, Russell LD, Friel PF, Griswold MD 2001 Murine germ cells do not require functional androgen receptors to complete spermatogenesis following spermatogonial stem cell transplantation. Endocrinology 142:2405-2408

Jones AR 1978 The antifertility actions of $\alpha$-chlorohydrin in the male. Life Sci 23:1625-1646

Jutte NHPM, Jansen R, Grootegoed JA, Rommerts FFG, van der Molen HJ 1983 FSH stimulation of the production of pyruvate and lactate by rat Sertoli cells may be involved in hormonal regulation of spermatogenesis. J Reprod Fertil 68:219-226

Kastner P, Mark M, Leid M, Gansmuller A, Chin W, Grondona JM, Decimo D, Krezel W, Dierich A, Chambon P 1996 Abnormal spermatogenesis in RXR $\beta$ mutant mice. Genes Dev 10:80-92

Kim KH, Akmal KM 1996 Role of vitamin A in male germ cell development. In: Desjardins C, ed. Cellular and Molecular Regulation of Testicular Cells. New York: Springer; 83-98

Kleene KC 1996 Patterns of translational regulation in the mammalian testis. Mol Reprod Devel 43:268-281

Kleene KC, Distel RJ, Hecht NB 1984 Translational regulation and deadenylation of a protamine mRNA during spermiogenesis in the mouse. Dev Biol 105:71-79

Knudson CM, Tung KSK, Tourtellotte WG, Brown GA, Korsmeyer SJ 1995 Bax-deficient mice with lymphoid hyperplasia and male germ cell death. Science 270:96-99

Korsmeyer SJ 1995 Regulators of cell death. Trends Genet 11:101-105

Kralewski M, Benavente R 1997 XY body formation during rat spermatogenesis: an immunocytochemical study using antibodies against XY body-associated proteins. Chromosoma 106: 304-307

Krishnamurthy H, Danilovich N, Morales CR, Sairam MR 2000 Qualitative and quantitative decline in spermatogenesis of the follicle-stimulating hormone receptor knockout (FORKO) mouse. Biol Reprod 62:1146-1159

Kumar TR, Wang Y, Lu N, Matzuk MM 1997 Follicle stimulating hormone is required for ovarian follicle maturation but not male fertility. Nature Genet 15:201-204

Li S, Zhou W, Doglio L, Goldberg E 1998 Transgenic mice demonstrate a testis-specific promoter for lactate dehydrogenase, LDHC. J Biol Chem 273:31191-31194

Liu F, Kondova I, Kilpatrick D 2000 Detection of PACH1, a nuclear factor implicated in the transcriptional regulation of meiotic and early haploid stages of spermatogenesis. Mol Reprod Dev 57:224-231

Lufkin T, Lohnes D, Mark M, Dierich M, Gorry P, Gaub M-P, LeMeur M, Chambon P 1993 High postnatal lethality and testis degeneration in retinoic acid receptor $\alpha$ mutant mice. Proc Natl Acad Sci USA 90:7225-7229

Maekawa M, O'Brien DA, Allen RL, Eddy EM 1989 Heat-shock cognate protein (hsc71) and related proteins in mouse spermatogenic cells. Biol Reprod 40:843-852

Mandal A, Naaby-Hansen S, Wolkowicz M, Klotz K, Shetty J, Retief J, Coonrod S, Kinter M, Sherman N, Cesar F, Flickinger C, Herr J 1999 FSP95, a testis-specific 95-kilodalton fibrous sheath antigen that undergoes tyrosine phosphorylation in capacitated human spermatozoa. Biol Reprod 61:1184-1197

Manova K, Nocka K, Besmer P, Bachvarova RF 1990 Gonadal expression of c-kit encoded at the $W$ locus of the mouse. Development 110:1057-1069 
Matsumoto M, Fujimoto H 1990 Cloning of a hsp70-related gene expressed in mouse spermatids. Biochem Biophys Res Comm 166:43-49

McCarrey JR 1994 Evolution of tissue-specific gene expression in mammals. BioScience 44:20-27

McCarrey JR, Thomas K 1987 Human testis-specific PGK gene lacks introns and possesses characteristics of a processed gene. Nature 326:501-505

Means AR, Cruzalegui F, LeMagueresse B, Needleman DS, Slaughter GR, Ono T 1991 A novel $\mathrm{Ca}^{2+} /$ calmodulin-dependent protein kinase and a male germ cell-specific calmodulin-binding protein are derived from the same gene. Mol Cell Biol 11:3960-3971

Meng X, Lindahl M, Hyvonen ME, Parvinen M, De Rooij DG, Hess MW, Raatikainen-Ahokas A, Sainio K, Rauvala H, Lakso M, Pichel JG, Weshphal H, Saarma M, Sariola H 2000 Regulation of cell fate decision of undifferentiated spermatogonia by GDNF. Science 287:1489-1493

Meuwissen RLJ, Offenberg HH, Dietrich AJJ, Riesewijk A, Van Iersel M, Heyting C 1992 A coiled-coil related protein specific for synapsed regions of meiotic prophase chromosomes. EMBO J 11:5091-5100

Mohri H, Mohri T, Ernster L 1965 Isolation and enzymatic properties of the midpiece of bull spermatozoa. Exp Cell Res 38:217-246

Mori C, Welch JE, Sakai Y, Eddy EM 1992 In situ localization of spermatogenic cell-specific glyceraldehyde 3-phosphate dehydrogenase (Gapd-s) messenger ribonucleic acid in mice. Biol Reprod 46:859-868

Mori C, Welch JE, Fulcher KD, O'Brien DA, Eddy EM 1993 Unique hexokinase messenger ribonucleic acids lacking the porin-binding domain are developmentally expressed in mouse spermatogenic cells. Biol Reprod 49:191-203

Mori C, Nakamura N, Welch JE, Gotoh H, Goulding EH, Fajioka M, Eddy EM 1998 Mouse spermatogenic-cell specific type 1 hexokinase $(m H k l-s)$ transcripts are expressed by alternative splicing from the $m H k 1$ gene and the HK1-S protein is localized mainly in the sperm tail. Mol Reprod Dev 49:374-385

Moss SB, VanScoy H, Gerton GL 1997 Mapping of a haploid transcribed and translated spermspecific gene to the mouse X chromosome. Mamm Genome 8:37-38

Nakamura M, Fujiwara A, Yasamasu I, Okinaga S, Arai K 1982 Regulation of glucose metabolism by adenine nucleotides in round spermatids from rat testes. $\mathrm{J}$ Biol Chem 257:13945-13950

O'Brien DA 1987 Stage-specific protein synthesis by isolated spermatogenic cells throughout meiosis and early spermiogenesis in the mouse. Biol Reprod 37:147-157

O'Brien DA, Gabel CA, Eddy EM 1993 Mouse Sertoli cells secrete mannose 6-phosphate containing glycoproteins that are endocytosed by spermatogenic cells. Biol Reprod 49:10551065

O'Brien DA, Welch JE, Fulcher KD, Eddy EM 1994 Expression of mannose 6-phosphate receptor messenger ribonucleic acids in mouse spermatogenic and Sertoli cells. Biol Reprod 50:429435

Pearse RVI, Drolet EW, Kalla KA, Hooshmand F, Bermingham JRJ, Rosenfeld MG 1997 Reduced fertility in mice deficient for the POU protein sperm-1. Proc Natl Acad Sci USA 94:7555-7560

Pittman DL, Cobb J, Schimenti KJ, Wilson LA, Cooper DM, Brugnull E, Handel MA, Schimenti JC 1998 Meiotic prophase arrest with failure of chromosome synapsis in mice deficient for Dmc1, a germline-specific RecA homolog. Mol Cell 1:697-705

Reddi PP, Flickinger CJ, Herr JC 1999 Round spermatid-specific transcription of the mouse SP-10 gene is mediated by a 294-base pair proximal promoter. Biol Reprod 61:1256-1266 
Reinke V, Smith H, Nance J, Wang J, Van Doren C, Begley R, Jones S, Davis E, Scherer S, Ward S, Kim S 2000 A global profile of germline gene expression in C. elegans. Mol Cell 6:605-616

Rodriguez I, Ody C, Araki K, Garcia I, Vassalli P 1997 An early and massive wave of germinal cell apoptosis is required for the development of functional spermatogenesis. EMBO J 9:2262-2270

Roosen-Runge EC 1977 The Process of Spermatogenesis. London: Cambridge University Press

Rosario MO, Perkins SL, O'Brien DA, Allen RL, Eddy EM 1992 Identification of the gene for the developmentally expressed $70 \mathrm{kDa}$ heat-shock protein (P70) of mouse spermatogenic cells. Dev Biol 150:1-11

Ross AJ, Waymire KG, Moss JE, Parlow AF, Skinner MK, Russell LD, MacGregor GR 1998 Testicular degeneration in Bclw-deficient mice. Nature Genet 18:251-256

Rossi P, Albanesi C, Grimaldi P, Geremia R 1991 Expression of the mRNA for the ligand of c-kit in mouse Sertoli cells. Biochem Biophys Res Commun 176:910-914

Sar M, Hall SH, Wilson EM, French FS 1993 Androgen regulation of Sertoli cells. In: Russell LD, Griswold MD, eds. The Sertoli Cell. Clearwater, FL: Cache River Press; 509-516

Sharpe RM 1994 Regulation of spermatogenesis. In: Knobil E, Neill J, eds. The Physiology of Reproduction. New York: Raven Press; 1363-1434

Skinner MK 1993 Secretion of growth factors and other regulatory factors. In: Russell LD, Griswold MD, eds. The Sertoli Cell. Clearwater, FL; Cache River Press; 237-247

Stevenson D, Jones AR 1985 Production of (S)-3-chloroactaldehyde from (S)-a-chlorohydrin by boar spermatozoa and the inhibition of glyceraldehyde-3-phosphate dehydrogenase in vitro. J Reprod Fertil 74:157-165

Storey BT, Kayne FJ 1978 Energy metabolism of spermatozoa. VII. Interactions between lactate, pyruvate and malate as oxidative substrates for rabbit sperm mitochondria. Biol Reprod 18:527-536

Syed V, Hecht NB 1997 Up-regulation and down-regulation of genes expressed in cocultures of rat Sertoli cells and germ cells. Mol Reprod Dev 47:380-389

Tapanainen JS, Aittomaki K, Mui J, Vaskivuo T, Huhtaniemi IT 1997 Men homozygous for an inactivating mutation of the follicle-stimulating hormone (FSH) receptor gene present variable suppression of spermatogenesis and fertility. Nature Genet 15:205-206

Tourtellotte W, Nagarajan J, Auyeung A, Mueller C, Milbrandt J 1999 Infertility associated with incomplete spermatogenic arrest and oligozoospermia in Efr4-deficient mice. Development 126:5061-5071

Travis AJ, Foster JA, Rosenbaum NA, Visconti PE, Gerton GL, Moss SB 1998 Targeting of a germ cell-specific type 1 hexokinase lacking a porin-binding domain to the mitochondria as well as to the head and fibrous sheath of murine spermatozoa. Mol Biol Cell 9:263-276

Tsunekawa N, Matsumoto M, Tone S, Nishida T, Fumimoto H 1999 The Hsp70 homolog gene, $H s c 70 t$, is expressed under translational control during mouse spermiogenesis. Mol Reprod Dev 52:383-391

Tsuruta JK, O'Brien DA 1995 Sertoli cell-spermatogenic cell interaction: the insulin-like growth factor-II/cation-independent mannose 6-phosphate receptor mediates changes in spermatogenic cell gene expression in mice. Biol Reprod 53:1454-1464

Tsuruta JK, Eddy EM, O'Brien DA 2000 Insulin-like growth factor-II/cation-independent mannose 6-phosphate receptor mediates paracrine interactions during spermatogonial development. Biol Reprod 63:1006-1013

Vidal F, Lopez P, Lopez-Fernandez LA, Ranc F, Scimeca JC, Cuzin F, Rassoulzadegan M 2001 Gene trap analysis of germ cell signaling to Sertoli cells: NGF-TrkA mediated induction of Fra1 and Fos by post-meiotic germ cells. J Cell Sci 114:435-443 
Vijayaraghavan S, Liberty G, Mohan J, Winfrey V, Olson G, Carr D 1999 Isolation and molecular characterization of AKAP110, a novel, sperm-specific protein kinase A-anchoring protein. Mol Endocrinol 13:705-717

Wang P, McCarrey JR, Yang F, Page DC 2001 An abundance of X-linked genes expressed in spermatogonia. Nature Genet 27:422-426

Wang Z, Kim KH 1993 Vitamin A-deficient testis germ cells are arrested at the end of S phase of the cell cycle: a molecular study of the origin of synchronous spermatogenesis in regenerated seminiferous tubules. Biol Reprod 48:1157-1165

Welch JE, Schatte EC, O'Brien DA, Eddy EM 1992 Expression of a glyceraldehyde 3-phosphate dehydrogenase gene specific to mouse spermatogenic cells. Biol Reprod 46:869-878

Welch JE, Brown PR, O'Brien DA, Eddy EM 1995 Genomic organization of a mouse glyceraldehyde 3-phosphate dehydrogenase gene (Gapd-s) expressed in post-meiotic spermatogenic cells. Dev Genet 16:179-189

Willison K, Ashworth A 1987 Mammalian spermatogenic gene expression. Trends Genet 3:351-355

Wolgemuth DJ, Watrin F 1991 List of cloned mouse genes with unique expression patterns during spermatogenesis. Mamm Genome 1:283-288

Yoshida K, Kondoh G, Matsuda Y, Habu T, Nishimune Y, Morita T 1998 The mouse RecA-like gene $\mathrm{Dmcl}$ is required for homologous chromosome synapsis during meiosis. Mol Cell 1:707-718

Yoshinaga K, Nishikawa S, Ogawa M, Hayashi S-I, Kunisada T, Fujimoto T, Nishikawa S-I 1991 Role of c-kit in mouse spermatogenesis: identification of spermatogonia as a specific site of c-kit expression and function. Development 113:689-699

Zabludoff S, Charron M, DeCerbo J, Simukova N, Wright W 2001 Male germ cells regulation transcription of the cathepsin 1 gene by rat Sertoli cells. Endocrinology 142:2318-2327

Zakeri ZF, Wolgemuth DJ, Hunt CR 1988 Identification and sequence analysis of a new member of the mouse HSP70 gene family and characterization of its unique cellular and developmental pattern of expression in the male germ line. Mol Cell Biol 8:2925-2932

Zhang L, Zhou W, Velculescu VE, Kern SE, Hruban RH, Hamilton SR, Vogelstein B, Kinzler KW 1997 Gene expression profiles in normal and cancer cells. Science 276:1268-1272

Zhao G-Q, Deng K, Labosky PA, Liaw L, Hogan BLM 1996 The gene encoding bone morphogenetic protein $8 \mathrm{~B}$ is required for the initiation and maintenance of spermatogenesis in the mouse. Genes Dev 10:1657-1669

Zhao G-Q, Liaw L, Hogan BLM 1998 Bone morphogenetic protein 8A plays a role in the maintenance of spermatogenesis and the integrity of the epididymis. Development 125:11031112

Zhu D, Dix DJ, Eddy EM 1997 HSP70-2 is required for CDC2 kinase activity in meiosis I of mouse spermatocytes. Development 124:3007-3014 